\title{
Effect of Bovine Asprosin on Gluconeogenic Gene Expression and Glucose Production in Primary Bovine Hepatocytes
}

\author{
Miao Lin \\ Yangzhou University \\ Maocheng Jiang \\ Yangzhou University \\ Tianyu Yang \\ Yangzhou University \\ Xiaoyu Ma \\ Yangzhou University \\ Guoqi Zhao \\ Yangzhou University \\ kang zhan ( $\nabla$ kzhan@yzu.edu.cn ) \\ Yangzhou University
}

\section{Research}

Keywords: Asprosin, hepatocytes, gluconeogenesis, glucose, postpartum

Posted Date: September 15th, 2021

DOI: https://doi.org/10.21203/rs.3.rs-880144/v1

License: (1) This work is licensed under a Creative Commons Attribution 4.0 International License. Read Full License 


\section{Abstract}

\section{Background}

In postpartum dairy cows, dry matter intake (DMI) was dramatically decreased, resulting in the decreased plasma glucose, a negative energy balance (NEB), and negative nutrient balance. Asprosin, which is a fasting-induced glucogenic protein hormone secreted by adipose tissue, promotes plasma glucose level. However, effect of asprosin on the hepatic glucose output in primary bovine hepatocytes and plasma asprosin level in postpartum dairy cows remain not reported.

Results

Our results demonstrated that fibrillin 1 (FBN1) showed much higher mRNA expression in mammary gland and adipose tissue compared with heart, liver, spleen, lung, and kidney. The recombinant bovine His-asprosin was not found in supernatant of E. coli lysate, but present in the inclusion bodies in E. coli. The bovine His-asprosin proteins were $>90 \%$ pure by Coomassie Blue-stained SDS-PAGE gel analysis. Asprosin enhanced $(P=0.031)$ the mRNA expression of PCK2 in primary bovine hepatocyte compared with control group, and FBP1 tended $(P=0.086)$ to be upregulated in primary bovine hepatocyte treated by Asprosin. Remarkably, glucose output was increased $(P=0.03)$ in primary bovine hepatocytes exposed to asprosin than control group. In addition, Asprosin can promote PKA activity in primary bovine hepatocytes, but not AKT. Asprosin was observed to be present in bovine plasma at consistent nanomolar levels. The postpartum cows exhibited $(P=0.003)$ much higher level of circulating asprosin compared with mid-lactation dairy cows.

\section{Conclusions}

These findings indicate that asprosin should be further considered for use as a novel therapy strategy for NEB and negative nutrient balance in postpartum period.

\section{Introduction}

During the transition to the lactation period, dairy cows experience a dramatic decrease in dry matter intake (DMI), triggering an attenuation in circulating glucose concentration, a negative energy balance (NEB) and negative nutrient balance [1, 2]. The subcutaneous adipose tissues mobilization is enhanced and lipogenesis is reduced in adipocytes due to the decreased circulating plasma insulin and glucose concentrations and increased concentrations of circulating catecholamines, growth hormone, and glucocorticoids [3] in postpartum cows. Therefore, plasma glucose and others metabolite are probably involved in the regulation of the hormone. In the current study, a major challenge in the field of postpartum dairy cows is the adequate elevation in the appetite and plasma glucose level, and maintaining the energy homeostasis. 
Asprosin, which is a C-terminal cleavage product of fibrillin 1 (FBN1) by furin protease, is a fastinginduced glucogenic protein hormone secreted by adipose tissue [4]. The circulating asprosin is recruited to the liver and binds to the G protein-coupled receptor OLFR734 to enhance the gluconeogenesis and hepatic glucose production via the activation of cAMP-PKA signaling pathway $[5,6]$. Previous study has reported that circulating asprosin crosses the blood-brain barrier to activate orexigenic AgRP neurons via a cAMP dependent pathway to stimulate appetite behaviors [7]. Additionally, asprosin can also regulate appetitive behaviors by OLFR734 [6]. In addition, asprosin promotes the expression of genes involved in the gluconeogenic genes G6PC and PCK1 and glucose production [5]. In ruminants, gluconeogenesis, which mainly occurs in liver, is one of the most important ways of supplying glucose requirements [8]. However, the mechanisms underlying the regulatory effects of asprosin on the gluconeogenic genes expression and glucose output remains unknown in primary bovine hepatocytes.

Therefore, objectives of this study was firstly to test this possibility, we determined in this study whether and where the FBN1 and furin were expressed in bovine tissues, and whether asprosin is in the circulating blood. Secondly, this study was to evaluate the effect of asprosin on gluconeogenic gene expression and glucose production in primary bovine hepatocytes, and provide a novel strategy for stimulating appetite behaviors, glucose production, and maintaining the relative energy balance in postpartum cows.

\section{Materials And Methods}

\section{Animals}

This study was carried out in accordance with the principles of Yangzhou University, the Institutional Animal Care and Use Committee (SYXK (Su) IACUC 2012-0029). All procedures involving the use of live bovine animals was approved by the Institutional Animal Care and Use Committee. Six Holstein cows were used to determine the expression of FBN1 and furin in different tissues. The early lactation (approximately $7 \mathrm{~d}$ after parturition) and mid-lactation Holstein cows were used to detect circulating asprosin in the blood. All cows were free of clinical signs of disease before isolation of the plasma. These cows were fed a TMR to meet $100 \%$ of NRC requirements. Cows were milked three times daily at 8:00, 14:00, and 21:00, respectively. The blood (approximately $5 \mathrm{~mL}$ ) was collected by venipuncture of the jugular vein from each cow before the morning feeding. These tubes were centrifuged at $1,000 \times \mathrm{g}$ at $4^{\circ} \mathrm{C}$ for $30 \mathrm{~min}$ to isolate the plasma. Plasma samples were immediately transported to laboratory and stored at $-80^{\circ} \mathrm{C}$ until the analysis of asprosin level.

\section{Expression evaluation of recombinant asprosin}

Bovine FBN1 (2732-2871 amino acids) cDNA was cloned and subsequently sub-cloned into a pET-30a vector using Nde I and Hind III for expression in E. coli. The fusion protein expressed in E. coli is 147 amino acids comprising of an amino-acid Met, a six-amino-acid His tag on the $\mathrm{N}$ terminus, and a 140amino-acid wild-type asprosin. The pET-30a vector containing 140-amino-acid asprosin was performed a transformation and incubated the plate upside down at $37^{\circ} \mathrm{C}$ overnight. Three single, well-isolated colonies were picked and inoculated it into $4 \mathrm{ml}$ LB medium containing $50 \mu \mathrm{g} / \mathrm{mL}$ kanamycin, 
respectively. When the OD600 value reaches $0.6 \sim 0.8$, two of the three tubes are added IPTG with the final concentration of $0.5 \mathrm{mM} \mathrm{IPTG}$ for induction at $15^{\circ} \mathrm{C}$ for $16 \mathrm{~h}$ and at $37^{\circ} \mathrm{C}$ for $4 \mathrm{~h}$, respectively, and the last one tube as the negative control. The protein expression and solubility were detected using both SDS-PAGE and western blot.

\section{Scale up expression, purification, and analysis of recombinant asprosin}

Cell pellets were resuspended with lysis buffer followed by sonication. The precipitate was dissolved using denaturing agent imidazole buffer. Target protein was obtained by two-step purification using $\mathrm{Ni}$ column and Superdex 200 column. Target protein was sterilized by $0.22 \mu \mathrm{m}$ filter before stored in aliquots. The concentration was determined by Bradford protein assay with BSA as standard. The protein purity and molecular weight were determined by standard SDS-PAGE along with Western blot.

\section{Cell culture}

Bovine hepatocytes were obtained as described previously [9] by the collagenase IV (Invitrogen, Shanghai, China) perfusion liver tissues collected from three nonlactating nongestating dairy cows at a local abattoir. The liver was perfused through vessels with perfusion solution A ( $140 \mathrm{mM} \mathrm{NaCl}, 10 \mathrm{mM} \mathrm{HEPES}$,

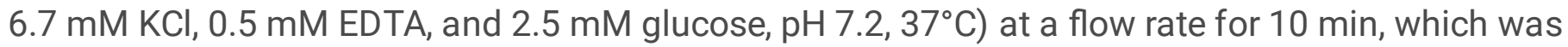
followed by solution $B(140 \mathrm{mM} \mathrm{NaCl}, 30 \mathrm{mM}$ HEPES, $6.7 \mathrm{mM} \mathrm{KCl}, 5 \mathrm{mM} \mathrm{CaCl} 2$, and $2.5 \mathrm{mM}$ glucose, $\mathrm{pH}$ $\left.7.2,37^{\circ} \mathrm{C}\right)$ at a flow rate for $10 \mathrm{~min}$. The collagenase IV solution $(0.1 \mathrm{~g}$ collagenase IV dissolved in $0.5 \mathrm{~L}$ of perfusion solution $\mathrm{B}, \mathrm{pH} 7.2,37^{\circ} \mathrm{C}$ ) was introduced to digest liver tissue. The collagenase digestion was terminated by addition of $50 \mathrm{~mL}$ fetal bovine serum (FBS; Gemini, Shanghai, China). The liver capsule, blood vessels, fat, and connective tissues were carefully removed, and the remainder of the liver parenchyma was minced and filtered successively through a $150 \mu \mathrm{m}$ mesh and a $75 \mu \mathrm{m}$ mesh, respectively. Hepatocytes were then washed twice with PBS at $4^{\circ} \mathrm{C}$, incuabed once with red blood cell lysis for 5 min and resuspended in DMEM/F12 medium supplemented with $10 \%$ FBS, $1 \mu \mathrm{M}$ insulin, $1 \mu \mathrm{M}$ dexamethasone, $100 \mathrm{U} / \mathrm{mL}$ penicillin, and $100 \mu \mathrm{g} / \mathrm{mL}$ streptomycin (Solarbio, Beijing, China).

\section{Quantitative RT-PCR}

For the expression of genes involved in gluconeogenic pathway in primary bovine hepatocytes exposed with asprosin, hepatocytes $\left(2 \times 10^{5}\right.$ cells/well) were seeded in 6-well plates and grown at $37^{\circ} \mathrm{C}, 5 \% \mathrm{CO}_{2}$. Cells were divided into two experimental groups as follows: 1 . Control, DMEM/F12 medium; 2. Asprosin treatment, DMEM/F12 medium containing $100 \mathrm{nmoL} / \mathrm{L}$ asprosin. All treatments were incubated for $6 \mathrm{~h}$. After incubation, total RNA was isolated from the cultured cells using a TRIzol kit (Tiangen, Beijing, China). Reverse transcription (RT) was performed using an RT Kit (Takara, Beijing, China). RT reaction mixtures contained $1 \mu \mathrm{g}$ total RNA and $1 \times$ PrimeScript RT Master Mix in a final volume of $20 \mu \mathrm{L}$, and reactions were performed for $15 \mathrm{~min}$ at $37^{\circ} \mathrm{C}$. Reverse transcriptase was inactivated by heating to $85^{\circ} \mathrm{C}$ for $5 \mathrm{~s}$. qRT-PCR assays were performed using SYBR ${ }^{\circledR}$ Premix Ex Taq ${ }^{\text {TM }}$ II Kit (Takara). The qRT-PCR reaction mixture contained $1 \times$ SYBR ${ }^{\circledR}$ Premix $\operatorname{Ex~Taq~}^{\mathrm{Tm}} \| \mathrm{I}, 0.4 \mu \mathrm{mol} / \mathrm{L}$ each forward and reverse primers, and $100 \mathrm{ng}$ cDNA templates in a final volume of $20 \mu \mathrm{L}$, and reactions were performed as follows: initial 
denaturation at $95^{\circ} \mathrm{C}$ for $30 \mathrm{~s}$, followed by 40 cycles at $95^{\circ} \mathrm{C}$ for $5 \mathrm{~s}$ and $60^{\circ} \mathrm{C}$ for $30 \mathrm{~s}$. Before the qRT-PCR for samples, the amplification efficiencies of all primers were determined by using standard dilution series. The primers listed in Table 1 are from the previous studies [9]. The relative expression of target genes was normalized by the geometric mean of the selected reference genes GAPDH and calculated using the $2^{-\triangle \triangle C T}$ method.

Table 1

Primers for real-time PCR analyses

\begin{tabular}{|c|c|c|c|}
\hline Gene & Primer sequence, $5^{\prime}$ to $3^{\prime}$ & Accession number & Source \\
\hline \multirow[t]{2}{*}{ PCK2 } & F: 5 TGACTGGGCAAGGGGAGCCG 3 & NM_001205594.1 & Zhang et al., 2016 [10] \\
\hline & R: 5 GGGGCCACCCCAAAGAAGCC 3 & & \\
\hline \multirow[t]{2}{*}{ PC } & F: 5 CCACGAGTTCTCCAACACCT 3 & NM_177946.4 & Zhang et al., 2016 [10] \\
\hline & R: 5 TTCTCCTCCAGCTCCTCGTA 3 & & \\
\hline \multirow[t]{2}{*}{ G6PC } & F: 5TGATGGACCAAGAAAGATCCAGG 3 & NM_001076124.2 & Zhang et al., 2016 [10] \\
\hline & R: 5TATGGATTGACCTCACTGGCCCTCTT 3 & & \\
\hline \multirow[t]{2}{*}{ FBP1 } & F: 5 ATAGAGAAGGCAGGAGGAAT 3 & NM_001034447 & Zhang et al., 2016 [10] \\
\hline & R: 5 CAGGAACTCAGTCACATCTT 3 & & \\
\hline \multirow[t]{2}{*}{ GAPDH } & F: 5 GGGTCATCATCTCTGCACCT 3 & NM_001034034 & Zhang et al., 2016 [10] \\
\hline & R: 5 GGTCATAAGTCCCTCCACGA 3 & & \\
\hline
\end{tabular}

\section{Glucose output}

For measurement of glucose output in primary bovine hepatocytes exposed with asprosin, hepatocytes (2 $\times 10^{5}$ cells/well) were seeded in 6-well plates and grown at $37^{\circ} \mathrm{C}, 5 \% \mathrm{CO}_{2}$. Cells were divided into two experimental groups as follows: 1 . Control, DMEM/F12 medium; 2 . Asprosin treatment, DMEM/F12 medium containing $100 \mathrm{nmoL} / \mathrm{L}$ asprosin. All treatments were incubated for $6 \mathrm{~h}$. After incubation, medium was then replaced with $1 \mathrm{~mL}$ of glucose-free DMEM and supplemented with $10 \mathrm{mM}$ lactate and $1 \mathrm{mM}$ sodium pyruvate. After incubation for another $2 \mathrm{~h}$, glucose level in the medium was determined by kit (Applygen, E1011, Beijing, China). Results were normalized to protein content.

\section{Western blotting analysis}

Cells were lysed to extract total protein in a RIPA lysis and extraction buffer (Thermo Scientific, Shanghai, China) containing $1 \times$ protease inhibitor cocktail (Thermo Scientific) and $1 \times$ phosphatase inhibitor cocktail tablets (Roche, Shanghai, China). Protein concentrations were determined using a BCA kit (Beyotime, Beijing, China). Equal amounts of protein lysates were fractionated by SDS-PAGE and transferred to nitrocellulose membranes (PALL, Shanghai, China). The membranes were blocked with $5 \%$ 
horse serum and then incubated with gentle shaking overnight at $4^{\circ} \mathrm{C}$, with the primary antibody plus $5 \%$ horse serum in Tris-buffered saline with Tween (TBS-T: $10 \mathrm{mM}$ Tris- $\mathrm{HCl}, \mathrm{pH}$ 7.5, $150 \mathrm{mM} \mathrm{NaCl}, 0.05 \%$ Tween 20). The following primary antibodies were obtained from Cell Signaling Technology (CST, Shanghai, China): GAPDH, AKT, and phosphorylated (p)-PKA (1: 1000; CST, Shanghai, China). The horseradish peroxidase (HRP)-conjugated secondary antibody used, included goat anti-rabbit IgG (1: 5000; CST). The target bands were detected using the Super Signal West Femto Maximum Sensitivity Substrate or Pierce ECL Plus Western Blotting Substrate (Thermo Scientific).

\section{Sandwich ELISA}

For the endogenous asprosin sandwich ELISA, a rabbit anti-asprosin polyclonal antibody (ab53076, Abcam) against asprosin amino acids 102-140 (amino acids 2832-2871 of human fibrillin 1) was used as the capture antibody, and a mouse anti-FBN1 monoclonal antibody (H00002200-M01, Abnova) against asprosin amino acids $41-140$ (amino acids $2772-2871$ of human fibrillin 1 ) was used as the detection antibody. An anti-mouse secondary antibody linked to HRP (1:5000; Nanjing Biogot) was used to generate a signal. Increasing amounts $(0,1.25,2.5,5,10,20$, and $40 \mathrm{nmoL} / \mathrm{L})$ of recombinant asprosin (which contains an $\mathrm{N}$-terminal His tag) were used to generate a standard curve for both assays.

\section{Statistical analysis}

The statistical analysis was tested by the independent sample t-test, using SPSS 19.0 software (SPSS Inc.; Chicago, IL, USA). $P<0.05$ were considered significant, and $<0.01$ were highly significant. Trends toward significance are discussed at $0.05<P<0.10$.

\section{Results}

\section{Expression of FBN1 and furin mRNA in bovine different tissues}

To confirm the expression of FBN1 and furin protease, FBN1 and furin protease mRNA expression profile was assessed across various metabolically important organs in bovine. Based on qRT-PCR analyses, both FBN1 and furin mRNA were expressed in various bovine tissues, including heart, liver, spleen, lung, kidney, mammary gland, haunch fat, abdominal fat, intestine fat, and kidney fat (Figure. 1). The FBN1 showed much higher mRNA expression in mammary gland and adipose tissue compared with heart, liver, spleen, lung, and kidney (Figure. 1). In addition, liver tissue displayed the highest furin mRNA expression (Figure. 1). The white adipose tissue demonstrated much higher mRNA expression compared with heart, spleen, lung, and kidney (Figure. 1). These results indicated that white adipose tissue and mammary gland may generate and secrete asprosin due to much higher expression level of FBN1.

\section{Expression evaluation of recombinant asprosin}

To investigate the circulating asprosin level in bovine plasma, bovine FBN1 (2732-2871 amino acids) was sub-cloned into a pET-30a vector for expression in E. coli. in order to obtain the bovine recombinant 
asprosin. Our results displayed that recombinant asprosin was not almost found in supernatant of cell lysate, but expressed in cell lysate by SDS-PAGE (Figure. 2A). In addition, His tag recombinant asprosin was detected in cell lysate using the anti-His antibody by Western blot, but slightly expressed in supernatant of cell lysate (Figure. 2B). This suggests that bovine recombinant asprosin was present in the inclusion bodies in $E$. coli. The purified bovine recombinant asprosin was found in scale up expression by SDS-PAGE (Figure. 2C). Moreover, the purified bovine recombinant asprosin can be detected using mouse anti-His antibody and mouse anti-FBN1 monoclonal antibody (against 2772-2871 amino acids immunogen) by Western blot, respectively (Figure. 2D). The purified bovine recombinant asprosin was estimated by the Coomassie Blue-stained SDS-PAGE gel analysis under reducing condition in order to determine the purity level. The bovine His-asprosin proteins used in all recombinant protein experiments were $>90 \%$ pure.

\section{Effect of asprosin on expression of gluconeogenic genes and glucose production}

To assess that whether asprosin can promote the mRNA expression of genes involved in the gluconeogenesis pathway and glucose production, these genes were investigated by qRT-PCR and hepatic glucose release into the medium was analyzed by Kit. Our results demonstrated that mRNA expression of glucose-6-phosphatase (G6PC) and pyruvate carboxylase (PC) were elevated in bovine hepatocyte treated by asprosin compared with control group, but have no significant difference (Figure. 3A). Remarkably, addition of asprosin enhanced the mRNA expression of phosphoenolpyruvate carboxykinase 2 (PCK2) in primary bovine hepatocyte compared with control group (Figure. 3A). In addition, fructose-1,6-bisphophatase (FBP1) tended to be upregulated in primary bovine hepatocyte treated by asprosin compared to control group (Figure. $3 \mathrm{~A}$ ). To demonstrate that whether asprosin can activate the PKA signal pathway, PKA activity was evaluated by immunoblotting with PKA substrate antibody in primary bovine hepatocyte. Our results showed that PKA activity was significantly elevated by asprosin stimulation using immunoblot of PKA substrate antibody, but have no profound change in AKT activity (Figure. 3B). More importantly, hepatic glucose release into the medium was significantly increased in primary bovine hepatocytes exposed to asprosin compared with control group.

\section{Plasma levels of asprosin in mid-lactation and postpartum dairy cows}

In postpartum cows, DMI was dramatically decreased, triggering an attenuation in circulating glucose concentration. Asprosin is a fasting-induced glucogenic protein hormone. To investigate circulating asprosin level in mid-lactation and postpartum dairy cows, plasma levels of asprosin should be investigated. To measure circulating asprosin levels, we developed a sandwich ELISA. We constructed a standard curve using recombinant asprosin with $0,1.25,2.5,5,10,20$, and $40 \mathrm{nmoL} / \mathrm{L}$ (Figure. 4A). The standard curve was used to calculate the asprosin level of bovine plasma. Asprosin was observed to be present in bovine plasma at consistent nanomolar levels (Figure. 4B), which is consistent with previous studies $[4,11]$. The result showed that postpartum cows exhibited much higher level of asprosin 
compared to mid-lactation cows (Figure. 4B). It is worth noting that circulating asprosin was not found or lower level in some mid-lactation cows. This suggests that increased circulating asprosin in postpartum dairy cows may promote the hepatic glucose production by gluconeogenic pathway to meet energy demand.

\section{Discussion}

Asprosin, which is a C-terminal cleavage product of FBN1 by furin protease, is a fasting-induced glucogenic protein hormone secreted by adipose tissue during fasting [4]. Asprosin promotes the hepatic glucose production $[4,5]$ and centrally acting orexigenic hormone that stimulates appetite behaviors in the hypothalamus $[6,7]$. However, effect of asprosin on the hepatic glucose level in primary bovine hepatocytes and circulating asprosin level in postpartum dairy cows remain not reported. It is well-known that DMI and circulating glucose level are critical role for energy balance and milk yield in postpartum dairy cows. Therefore, recombinant bovine asprosin should firstly be obtained by prokaryotic expression system in vitro.

To determine that whether bovine can secret the asprosin, which is a C-terminal cleavage product of FBN1 by furin protease, we firstly detect the expression of FBN1 and furin protease in various metabolically important organs tissues. The bovine mammary gland and adipose tissues displayed much higher expression level in FBN1 compared with heart, liver, spleen, lung, and kidney. The furin protease is highest expression in bovine liver tissues, and white adipose tissues demonstrated much higher mRNA expression compared with heart, spleen, lung, mammary gland, and kidney. Previous study has also demonstrated that white adipose tissues is one of the sources of plasma circulating asprosin [4]. It is well-known that adipose acts as an endocrine organ and a regulator of energy homeostasis, which is consistent with the asprosin secreted by adipose tissues. Remarkably, mammary gland organ also could be one of the sources of plasma circulating asprosin, given much higher expression of FBN1 in mammary gland, and bovine mammary gland also acts as an important endocrine organ.

Asprosin functions as the increase in plasma glucose concentration [4]. Gluconeogenesis, which mainly occurs in liver, is one of the most important ways of supplying glucose requirements in postpartum cows. To investigate whether asprosin affects expression of genes involved in hepatic gluconeogenesis, we detected the key expression of genes in primary bovine hepatocyte, including four rate-limiting enzymes G6PC, FBP1, PC, and PCK [12]. We found that asprosin enhanced the mRNA expression of PCK2 in bovine hepatocyte compared with control group. In addition, FBP1 tended to be upregulated in bovine hepatocyte treated by asprosin. More importantly, glucose output was increased in bovine hepatocytes exposed to asprosin than control group. Previous studies mainly focus on the effect of propionate-induced gluconeogenesis $[9,13]$. The proportion of propionate involved in gluconeogenesis is between $50 \%$ and $60 \%$ in bovine hepatocytes $[14,15]$. In present study, asprosin has a profound change on the genes involved in gluconeogenesis and glucose out in primary bovine hepatocytes, suggesting that asprosin may respond to different gluconeogenic precursor to promote hepatic glucose production. Asprosin activates OLFR734 receptor to induce glucose output via cAMP-PKA second-messenger pathway [5]. Our 
result showed that PKA activity was evaluated in bovine hepatocyte induced by asprosin, but AKT activity was not altered. These results demonstrated that asprosin induce glucose output by PKA secondmessenger pathway.

Since the circulating asprosin level are increased by fasting [4]. The DMI were dramatically decreased in postpartum cows. We hypothesized that decreased DMI in postpartum period could induce the plasma asprosin level. To demonstrate this, we selected the mid-lactation cows and postpartum period cows to detect the circulating asprosin levels and found that postpartum period dairy cows displayed much higher level of asprosin compared to mid-lactation cows. The circulating asprosin level was decreased by increased feeding supply [4]. In mid-lactation dairy cows showed the high DMI and high or physiological glucose condition to inhibit the asprosin production, which is consistent with previous study that glucose acts as a negative regulatory factor of plasma asprosin levels in a negative-feedback loop [4]. Previous study has reported that circulating asprosin crosses the blood-brain barrier to activate orexigenic AgRP neurons via a cAMP dependent pathway to stimulate appetite behaviors [7]. Additionally, OLFR734 in the nervous system, which acts as a receptor of asprosin, stimulate the appetitive behaviors by improving olfactory performance [6]. During postpartum period cows, DMI was dramatically decreased, resulting in an attenuation in plasma glucose level. In current study, a major challenge in the field of postpartum dairy cattle is the adequate to increase the $\mathrm{DMI}$ and circulating glucose level, maintaining energy homeostasis. Therefore, asprosin should be further considered for use as a novel therapy strategy for NEB and energy homeostasis in postpartum period.

\section{Conclusion}

The present study demonstrated that mammary gland and adipose tissue displayed much higher expression of FBN1 mRNA, indicating that mammary gland could be one of the sources of plasma circulating asprosin. Expression of gluconeogenic genes (PCK2 and FBP1) and glucose output were elevated in hepatocytes by asprosin treatment. Importantly, postpartum cows exhibited much higher level of asprosin compared to mid-lactation dairy cows, suggesting that increased circulating asprosin may promote the plasma glucose level in postpartum cows. Therefore, asprosin should be further considered for use as a novel therapy strategy for NEB and energy homeostasis in postpartum period, which is important significance in milk production performance for early-lactation period and overall lactation period.

\section{Declarations}

\section{Declarations}

\section{Ethics approval and consent to participate}

The experiments were approved by the Institutional Animal Care and Use Committee of Yang Zhou University. 


\section{Consent for publication}

Not applicable.

\section{Competing interests}

The authors declare that they have no competing interests.

\section{Funding}

This study was supported by the National Natural Science Foundation of China (No. 32002200), the Research Project of Natural Science Foundation of Jiangsu Province (BK20190898), and China Agriculture Research System of MOF and MARA.

\section{Authors' contributions}

LM performed experiment work, analyzed the data, and wrote the manuscript. JMC, YTY, and MXY also performed experiment work. ZGQ revised the manuscript. ZK contributes to the experimental idea.

\section{References}

1. Zhang Q, Su HW, Wang FW, Cao ZJ, Li SL. Effects of energy density in close-up diets and postpartum supplementation of extruded full-fat soybean on lactation performance and metabolic and hormonal status of dairy cows. J Dairy Sci. 2015;98(10):7115-30.

2. Contreras GA, Strieder-Barboza C, Koster JD. Symposium review: Modulating adipose tissue lipolysis and remodeling to improve immune function during the transition period and early lactation of dairy cows. J Dairy Sci. 2018;101(3):2737-2752.

3. Bauman DE, Currie WB. Partitioning of nutrients during pregnancy and lactation: a review of mechanisms involving homeostasis and homeorhesis. J Dairy Sci. 1980;63(9): 1514-29.

4. Romere C, Duerrschmid C, Bournat J, Constable P, Jain M, Xia, et al. Asprosin, a Fasting-Induced Glucogenic Protein Hormone. Cell. 2016;165(3):566-79.

5. Li E, Shan H, Chen LQ, Long AJ, Zhang YY, Liu Y, et al. OLFR734 Mediates Glucose Metabolism as a Receptor of Asprosin. Cell Metab. 2019;30(2):319-328.

6. Liu Y, Long AJ, Chen LQ, Jia LJ, Wang YG. The Asprosin-OLFR734 module regulates appetitive behaviors. Cell Discov. 2020;6(1):19.

7. Duerrschmid C, He Y, Wang C, Li C, Bournat JC, Romere C, et al. Asprosin is a centrally acting orexigenic hormone. Nat Med. 2017;23(12):1444-53. 
8. Greenfield RB, Cecava MJ, Donkin SS. Changes in mRNA expression for gluconeogenic enzymes in liver of dairy cattle during the transition to lactation. J Dairy Sci. 2000;83(6):1228-36.

9. Liu L, Xing DG, Du XL, Peng T, McFadden W, Wen LX, et al. Sirtuin 3 improves fatty acid metabolism in response to high nonesterified fatty acids in calf hepatocytes by modulating gene expression. $J$ Dairy Sci. 2020;103(7): 6557-68.

10. Zhang Q, Koser SL, Donkin SS. Propionate induces mRNA expression of gluconeogenic genes in bovine calf hepatocytes. J Dairy Sci. 2016;99(5):3908-15.

11. Wang CY, Lin T, Liu KH, Liao CH, Liu YY, Wu VC, et al. Serum asprosin levels and bariatric surgery outcomes in obese adults. Int J Obes. 2019;43(5):1019-25.

12. Lin HV, Accili D. Hormonal regulation of hepatic glucose production in health and disease. Cell Metab. 2011;14(1):9-19.

13. Zhan $\mathrm{K}$, Yang TY, Chen YY, Jiang MC, Zhao GQ, et al. Propionate enhances the expression of key genes involved in gluconeogenic pathway in bovine intestinal epithelial cells. J Dairy Sci. 2020;103(6):5514-24.

14. Huntington, G. B. Energy metabolism in the digestive tract and liver of cattle: influence of physiological state and nutrition. Reprod Nutr Dev. 1990;30(1):35-47.

15. Aschenbach JR, Kristensen NB, Donkin SS, Hammon HM, Penner GB. Gluconeogenesis in dairy cows: the secret of making sweet milk from sour dough. Lubmb Life. 2010;62(12):869-77.

\section{Figures}
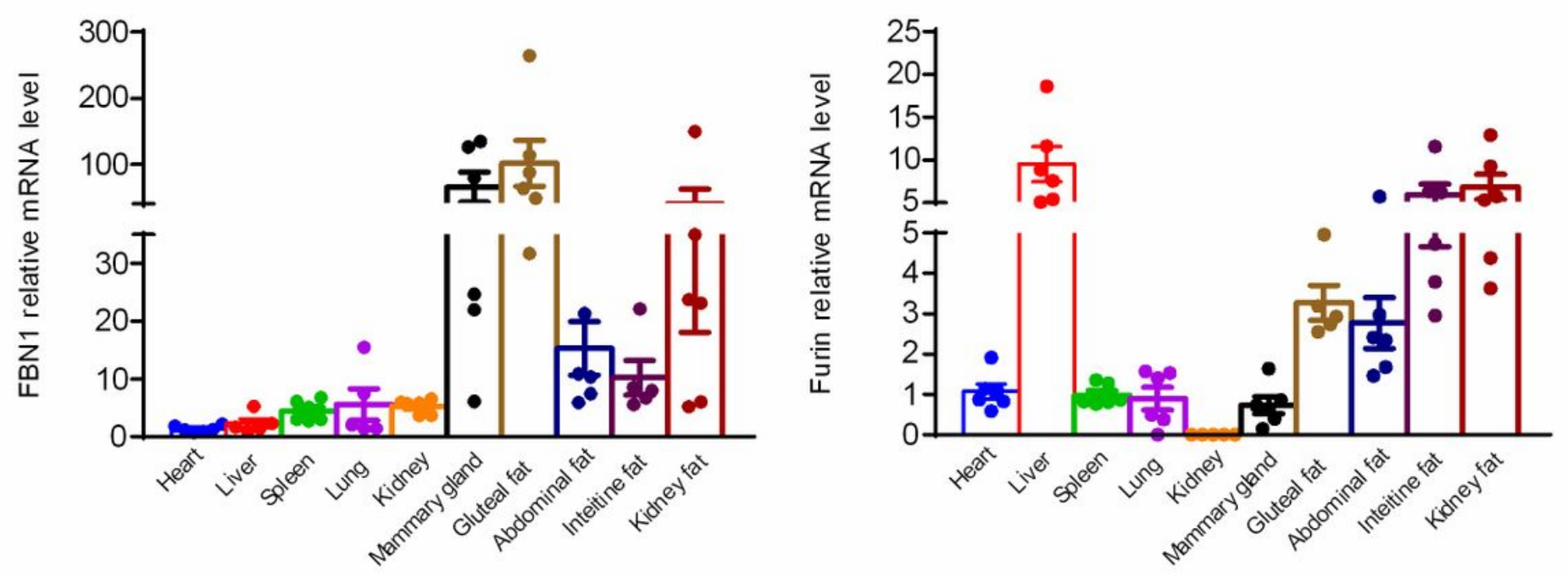
Figure 1

Expression of FBN1 and furin mRNA in bovine different tissues by qRT-PCR $(n=6)$.

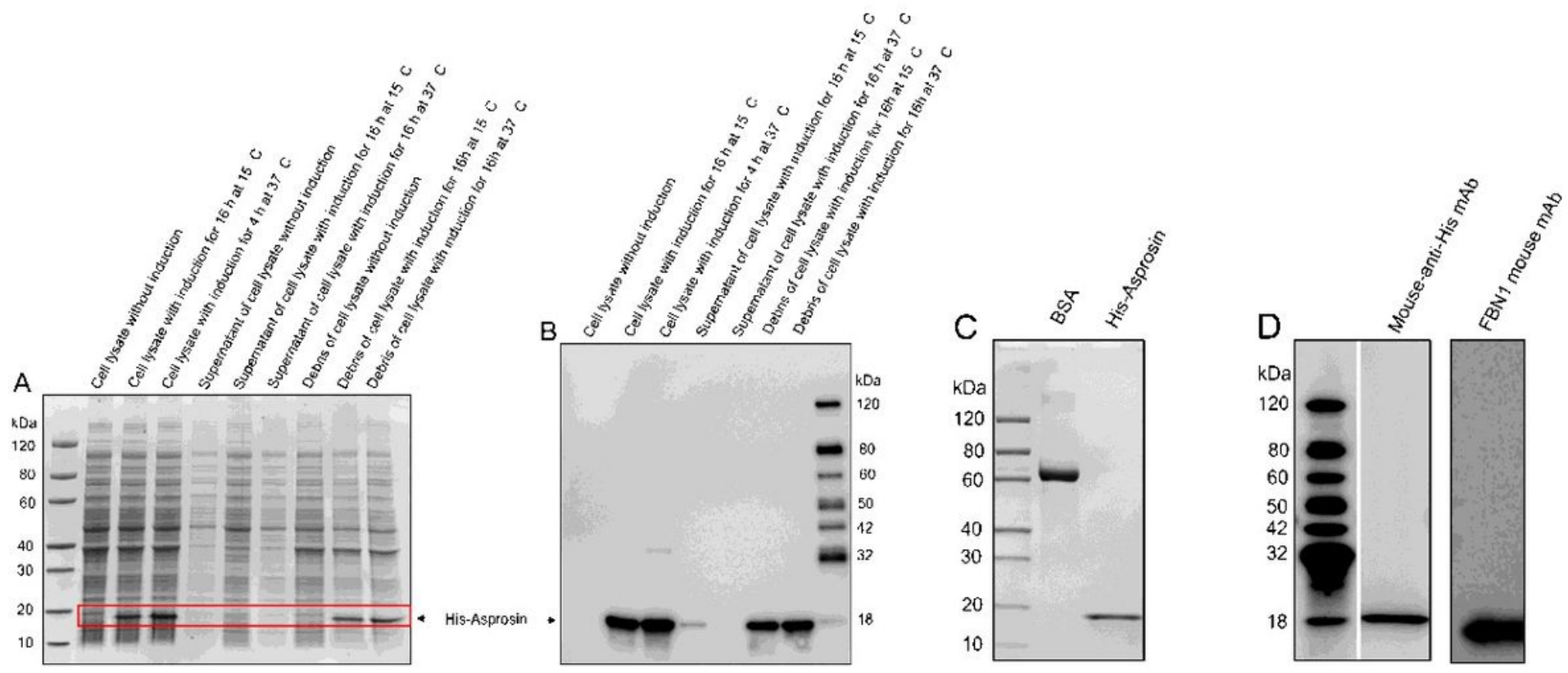

Figure 2

Expression evaluation of bovine recombinant asprosin and scale up expression. (A) SDS-PAGE analysis for asprosin cloned in pET-30a and expressed in BL21 (DE3) strain in different conditions. (B) Western blot analysis for protein lysate sample from different treated conditions using anti-His antibody. (C) SDSPAGE analysis for purified bovine recombinant asprosin by scale up expression. (D) Western blot analysis for purified bovine recombinant asprosin using mouse anti-His antibody and mouse anti-FBN1 monoclonal antibody (against 2772-2871 amino acids immunogen). 


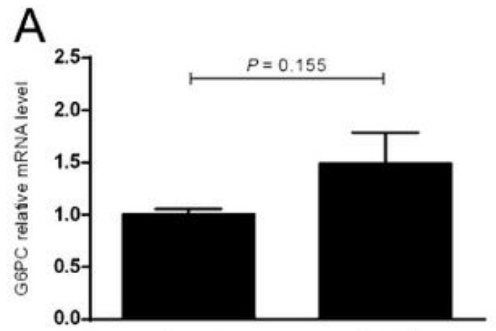

Control

Asprosin

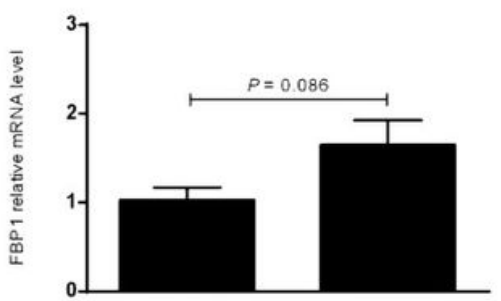

Control

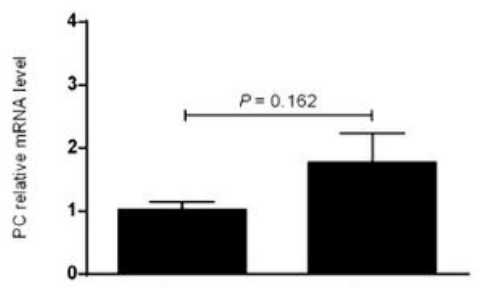

Control

Asprosin

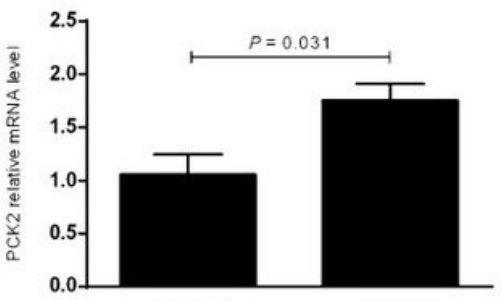

Control

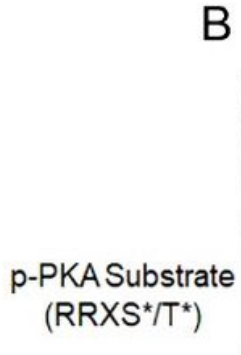

$B$

Control Asprosin

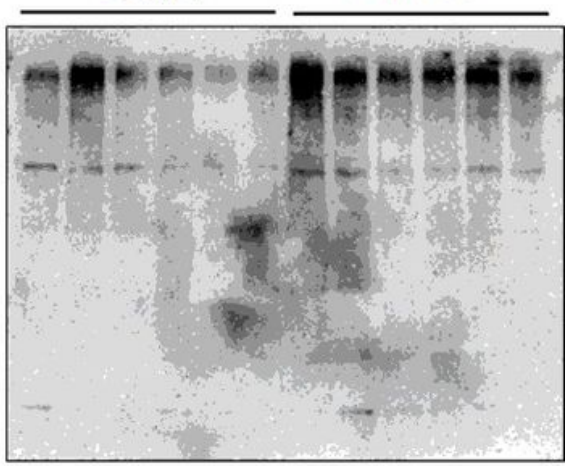

AKT

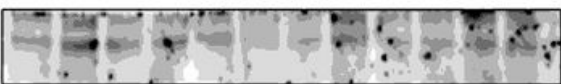

GAPDH

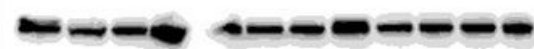

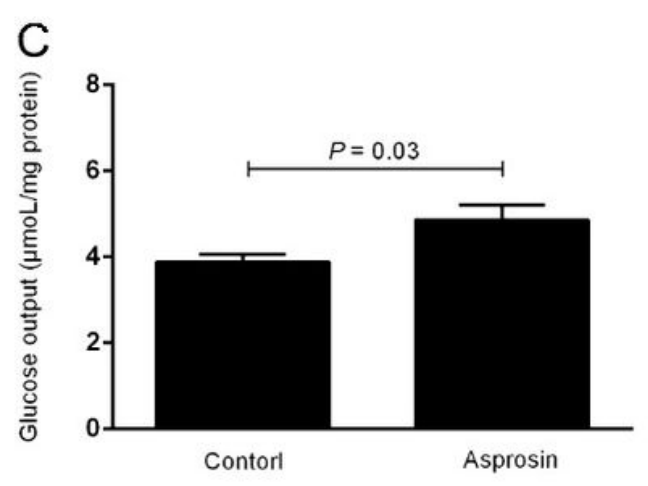

\section{Figure 3}

Asprosin enhances glucose production via activation of p-PKA in primary bovine hepatocytes. (A) qRTPCR analysis for expression of gluconeogenic genes $(n=4)$. (B) Western blot analysis for PKA activity using immunoblot of PKA substrate antibody $(n=6)$. (C) Effect of asprosin on glucose output in primary bovine hepatocytes $(n=6)$. 

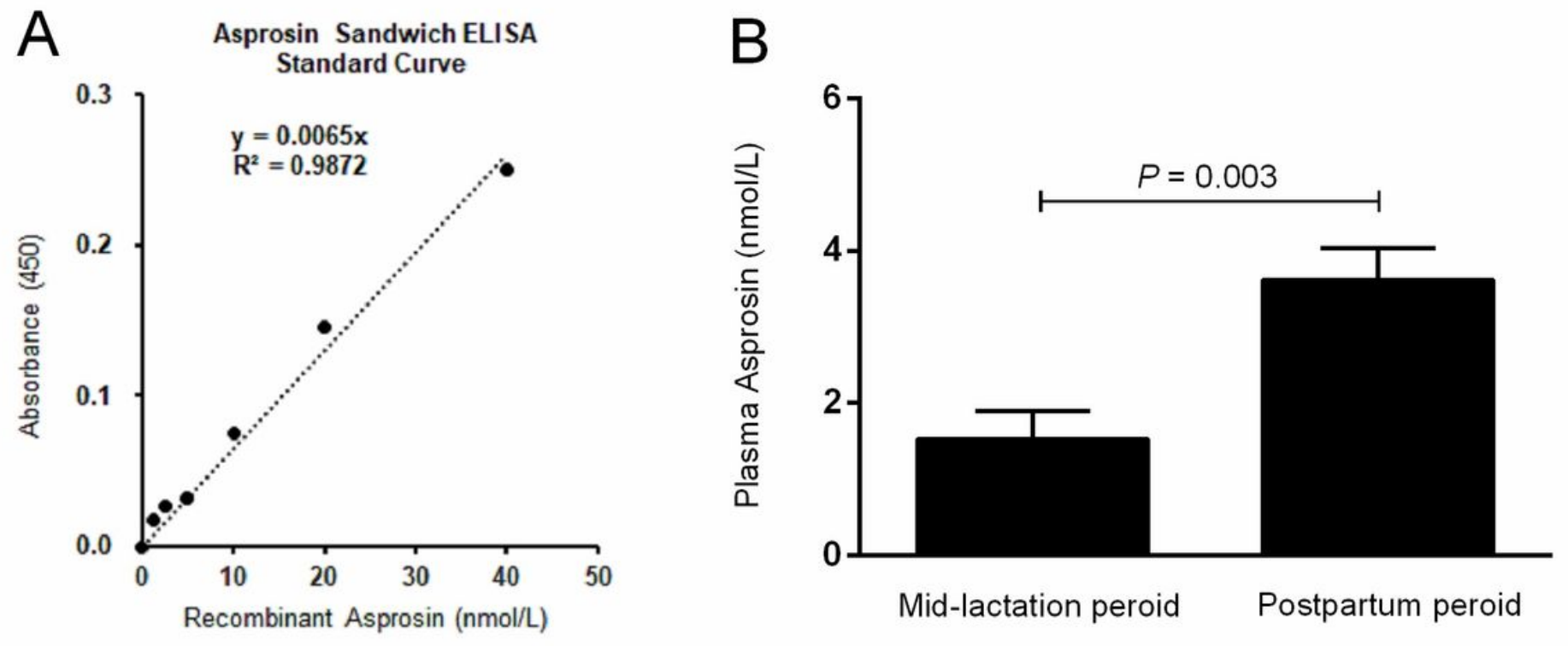

Figure 4

Effect of asprosin on the plasma levels of asprosin in mid-lactation and postpartum dairy cows. (A) Asprosin sandwich ELISA standard curve constract. (B) Sandwich ELISA was used to detect plasma circulating asprosin levels in mid-lactation and postpartum dairy cows $(n=12)$. 